K. TANUMA

KODAI MATH. J.

13 (1990), 155-163

\title{
REMARKS ON EFFECTIVE CURVATURE
}

\author{
By KaZUMI TANUMA
}

\section{Introduction}

Let $\Omega$ be a domain in $R_{x, y}^{2}$ with a reflecting smooth boundary $\Gamma$. Suppose that $\Omega$ is a media through which wave propagates with a speed $c(x, y)$. Let $s$ be the arc length of $\Gamma$ measured along the curve from a fixed point on $\Gamma$, and $n$ be the normal distance from $\Gamma$ to the point in $\bar{\Omega}$ such that internal points of $\Omega$ correspond to $n>0$. Now we suppose the center of curvature is in $\Omega$. Let $K_{0}(s)$ be the curvature of $\Gamma$ at $s$. Let the speed of wave propagation be constant. Then along a concave part of $\Gamma\left(K_{0}(s)>0\right)$, a high frequency wave well known by the name of whispering gallery wave can propagate. When the speed is variable, the role of the boundary curvature $K_{0}(s)$ should be replaced by the effective curvature $K(s)$.

Babich and Kirpichnikova [1] defined the effective curvature $K(s)$ by

$$
K(s)=K_{0}(s)+\left.c^{-1}(s, n) \partial_{n} c(s, n)\right|_{n=0} .
$$

Let $\omega$ be the frequency of wave and $L_{\varepsilon}$ be the boundary layer given by

$$
L_{\varepsilon}=\{(s, n) \in \Omega ; K(s)>\varepsilon>0, n \geqq 0\}
$$

where $n$ is sufficiently small.

They constructed a solution $U$ which satisfies the following Helmholtz equation asymptotically as $\omega \rightarrow+\infty$

$$
\left(\Delta_{x, y}+\omega^{2} c^{-2}(x, y)\right) U=0 \quad \text { in } L_{\varepsilon}
$$

with the Dirichlet boundary condition

$$
\left.U\right|_{\Gamma}=0
$$

such that the solution is concentrated near $\Gamma$ in the sense that

$$
U \longrightarrow 0 \quad \text { exponentially as } n \rightarrow+\infty \text {. }
$$

Let

$$
A i(x)=\int_{0}^{\infty} \cos \left(t^{3} / 3+x t\right) d t \quad(x \in R)
$$

Received June 14, 1989. 
be one of the Airy functions which is rapidly decreasing as $x \rightarrow+\infty$ together with all of its derivatives and has the zeros only on the negative real axis and let $-\nu<0$ be one of zeros of $A i(x)$. Then $U$ is given in the following form

$$
U(s, n) \sim \exp \left\{i \omega \int_{s_{0}}^{s} c^{-1}(s, 0) d s+i \omega^{1 / 3} h(s)\right\} \sum_{k=0}^{\infty} \omega^{-3 / k} U_{k}(s, n)
$$

where

and

$$
\begin{aligned}
& h(s)=-\nu \int_{s_{0}}^{s} K^{2 / 3}(s)\{2 c(s, 0)\}^{-1 / 3} d s \\
& U_{0}=\{2 K(s) c(s, 0)\}^{1 / 6} A i\left(\omega^{2 / 3} n \eta(s)-\nu\right) \\
& \eta(s)=\{2 K(s)\}^{1 / 3} c^{-2 / 3}(s, 0)
\end{aligned}
$$

$$
s_{0} \in \Gamma \quad \text { with } K\left(s_{0}\right)>\varepsilon .
$$

$U_{k}(k \geqq 1)$ can be obtained successively by solving a certain recursive equation. (see pp. $37 \sim 47$ in [1])

Let us assume all the rays of waves are tangent to $\Gamma$. Then in this paper we shall prove the effective curvature in (1.1) is simply given by the curvature of $\Gamma$ minus the ray curvature at the point of tangency, and also give the transformation invariant formula for the ray curvature in the two dimensional Riemannian space.

\section{Lemma and Main Theorem}

The eikonal equation for (1.2) is given by

$$
H(x y p q \tau)=(1 / 2)\left\{c^{2}\left(p^{2}+q^{2}\right)-1\right\}=0 \quad\left(p=\partial_{x} \tau, q=\partial_{y} \tau\right)
$$

where $\tau(x, y)$ is the phase function of the wave. Also the system of differential equations for the characteristics (i.e., rays) of (2.1) has the form

$$
\begin{aligned}
& \dot{x}=\partial H / \partial p=c^{2} p \quad \dot{y}=\partial H / \partial q=c^{2} q \\
& \dot{p}=-\partial H / \partial x=-c \partial_{x} c\left(p^{2}+q^{2}\right)=-(1 / c) \partial_{x} c \\
& \dot{q}=-\partial H / \partial y=-c \partial_{y} c\left(p^{2}+q^{2}\right)=-(1 / c) \partial_{y} c \\
& \dot{\tau}=p(\partial H / \partial p)+q(\partial H / \partial q)=c^{2}\left(p^{2}+q^{2}\right)=1
\end{aligned}
$$

where denotes a derivative with respect to a parameter of the ray.

LEMMA. Assume all the rays are tangent to $\Gamma$ and let $K_{r}(s)$ be the curvature of the ray at $s$ the point of tangency. Then the effective curvature (1.1) is given by

$$
K(s)=K_{0}(s)-K_{r}(s)
$$


Proof. It is enough to show

$$
K_{r}(s)=-\partial_{n} c(s, n) /\left.c(s, n)\right|_{n=0} .
$$

Let the generic points of $\Gamma$ be $(u(s), v(s))$ parametrized by the arc length $s$, and let each point $(x, y) \in \Omega$ near $\Gamma$ have the representation:

$$
x=u(s)-n v^{\prime}(s), \quad y=v(s)+n u^{\prime}(s) .
$$

Then the boundary curvature is given by

$$
K_{0}(s)=u^{\prime}(s) v^{\prime \prime}(s)-u^{\prime \prime}(s) v^{\prime}(s) .
$$

Let the ray parameter in (2.2) increase with $s$. Then taking the center of curvature in $\Omega$, we have the ray curvature

$$
K_{r}=(x \ddot{y}-\ddot{x} \dot{y}) \cdot\left(\dot{x}^{2}+\dot{y}^{2}\right)^{-3 / 2} .
$$

From $(2.2)$

$$
\begin{aligned}
& \ddot{x}=\left(c^{2} p\right)^{\cdot}=2 c\left\{\left(\partial_{x} c\right) \dot{x}+\left(\partial_{y} c\right) \dot{y}\right\} p+c^{2} \dot{p}=2 c^{3}\left\{\left(\partial_{x} c\right) p^{2}+\left(\partial_{y} c\right) p q\right\}-c \partial_{x} c \\
& \ddot{y}=\left(c^{2} q\right)^{\cdot}=2 c\left\{\left(\partial_{x} c\right) \dot{x}+\left(\partial_{y} c\right) \dot{y}\right\} q+c^{2} \dot{q}=2 c^{3}\left\{\left(\partial_{x} c\right) p q+\left(\partial_{y} c\right) q^{2}\right\}-c \partial_{y} c .
\end{aligned}
$$

Hence substituting them into $K_{r}$, we obtain

$$
K_{r}=\left\{c^{3}\left(\partial_{x} c\right) q-c^{3}\left(\partial_{y} c\right) p\right\}\left\{c^{4}\left(p^{2}+q^{2}\right)\right\}^{-3 / 2}=\left(\partial_{x} c\right) q-\left(\partial_{y} c\right) p .
$$

From (2.4) it can be easily seen that

$$
\begin{aligned}
\partial_{s} & =\left(u^{\prime}(s)-n v^{\prime \prime}(s)\right) \partial_{x}+\left(v^{\prime}(s)+n u^{\prime \prime}(s)\right) \partial_{y} \\
& =u^{\prime}(s)\left(1-n K_{0}(S)\right) \partial_{x}+v^{\prime}(s)\left(1-n K_{0}(S)\right) \partial_{y} \\
\partial_{n} & =-v^{\prime}(s) \partial_{x}+u^{\prime}(s) \partial_{y} .
\end{aligned}
$$

Here we have used the relations

$$
u^{\prime \prime}(s)=-K_{0}(s) v^{\prime}(s), \quad v^{\prime \prime}(s)=K_{0}(s) u^{\prime}(s),
$$

which can be derived from (2.5) and $u^{\prime \prime}(s) u^{\prime}(s)+v^{\prime \prime}(s) v^{\prime}(s)=0$. From (2.6) it follows immediately

$$
\begin{aligned}
& \partial_{x}=\left(1-n K_{0}(s)\right)^{-1} u^{\prime}(s) \partial_{s}-v^{\prime}(s) \partial_{n} \\
& \partial_{y}=\left(1-n K_{0}(s)\right)^{-1} v^{\prime}(s) \partial_{s}+u^{\prime}(s) \partial_{n}
\end{aligned}
$$

Therefore we obtain

$$
\begin{aligned}
K_{r}= & \left(\partial_{x} c\right)\left(\partial_{y} \tau\right)-\left(\partial_{y} c\right)\left(\partial_{x} \tau\right) \\
= & \left(1-n K_{0}(s)\right)^{-1}\left\{-\left(u^{\prime}(s)\right)^{2}-\left(v^{\prime}(s)\right)^{2}\right\}\left(\partial_{n} c\right)\left(\partial_{s} \tau\right) \\
& +\left(1-n K_{0}(s)\right)^{-1}\left\{\left(u^{\prime}(s)\right)^{2}+\left(v^{\prime}(s)\right)^{2}\right\}\left(\partial_{s} c\right)\left(\partial_{n} \tau\right) \\
= & -\left(1-n K_{0}(s)\right)^{-1}\left(\partial_{n} c\right)\left(\partial_{s} \tau\right)+\left(1-n K_{0}(S)\right)^{-1}\left(\partial_{s} c\right)\left(\partial_{n} \tau\right) .
\end{aligned}
$$


On the other hand, since the ray is tangent to $\Gamma$, it holds that $-v^{\prime}(s) \partial_{x} \tau+$ $u^{\prime}(s) \partial_{y} \tau=0$ at the point of tangency, that is, from (2.6)

$$
\partial_{n} \tau=0 \quad \text { at } n=0 \text {. }
$$

By (2.7) the eikonal equation (2.1) becomes

$$
c^{-2}=\left(1-n K_{0}(s)\right)^{-2}\left(\partial_{s} \tau\right)^{2}+\left(\partial_{n} \tau\right)^{2},
$$

which implies

$$
c^{-2}=\left(\partial_{s} \tau\right)^{2} \quad \text { at } \quad n=0 .
$$

Since $\dot{\tau}=1$ and $s$ increases with ray parameter, so does the phase $\tau$ along $s$.

Thus we have $\partial_{s} \tau>0$ and $\partial_{s} \tau=c^{-1}$ at $n=0$, which give $K_{r}(s)=-\partial_{n} c(s, n) /$ $\left.c(s, n)\right|_{n=0}$, and the proof is completed.

Q.E.D.

Next we consider the problem (1.2), (1.3), (1.4) in the domain $\Omega$ in two dimensional Riemannian space, so the Laplacian $\Delta$ in (1.2) should be replaced by the Laplace-Beltrami operator.

Let the fundamental tensor be $g_{\imath \jmath}, i, j=1,2$ (symmetric and positive definite), and the coordinate system be $u^{\imath}, i=1,2$, and let $g=\operatorname{det}\left(g_{\imath j}\right)$ and $\left(g^{\imath j}\right)=\left(g_{\imath j}\right)^{-1}$. In the following calculus, we follow Einstein's summation convention.

Now we put

$$
g_{\imath \jmath}=\left(\partial x^{1} / \partial u^{\imath}\right)\left(\partial x^{1} / \partial u^{j}\right)+\left(\partial x^{2} / \partial u^{\imath}\right)\left(\partial x^{2} / \partial u^{j}\right)
$$

where $\left(x^{1}, x^{2}\right)$ is the orthogonal coordinate and $\left\{e_{1}, e_{2}\right\}$, defined by

$$
e_{1}=\left(\partial x^{1} / \partial u^{1}, \partial x^{2} / \partial u^{1}\right), \quad e_{2}=\left(\partial x^{1} / \partial u^{2}, \partial x^{2} / \partial u^{2}\right),
$$

is taken as the natural base for the curvilinear coordinate system $\left(u^{2}\right)$.

Now making the substitution of $\Delta=g^{-1 / 2} \partial\left(g^{1 / 2} g^{\imath j} \partial / \partial u^{j}\right) / \partial u^{2}$ into (1.2) gives the equation

$$
\begin{aligned}
\left(\Delta+\omega^{2} c^{-2}\right) U= & g^{2 \jmath}\left(\partial^{2} U / \partial u^{2} \partial u^{j}\right)+\left(\partial g^{\imath \jmath} / u^{2}\right)\left(\partial U / \partial u^{j}\right) \\
& +g^{-1 / 2}\left(\partial g^{1 / 2} / \partial u^{i}\right) g^{\imath j}\left(\partial U / \partial u^{j}\right)+\omega^{2} c^{-2} U=0 .
\end{aligned}
$$

Here we assume that the leading term in the asymptotic solution $U$ is given in the form

$$
\left\{\exp i \omega \tau\left(u^{1} u^{2}\right)\right\} \varphi\left(u^{1} u^{2}\right) .
$$

So substituting this into (2.8) and collecting powers of $\omega^{2}$, we obtain

$$
\left\{-g^{\imath j}\left(\partial \tau / \partial u^{2}\right)\left(\partial \tau / \partial u^{j}\right)+c^{-2}\right\} \omega^{2}(\exp i \omega \tau) \varphi=0 .
$$

In this case the eikonal equation is defined as

$$
H\left(\begin{array}{lllll}
u^{1} & u^{2} & p_{1} & p_{2} & \tau
\end{array}\right)=(1 / 2)\left(c^{2} g^{\imath \jmath} p_{i} p_{j}-1\right)=0
$$

where $p_{i}=\partial \tau / \partial u^{2} i=1,2$. Thus the rays are obtained by solving 


$$
\begin{aligned}
& \dot{u}^{1}=\partial H / \partial p_{1}=c^{2} g^{1} p_{i} \quad \dot{u}^{2}=\partial H / \partial p_{2}=c^{2} g^{2 \jmath} p_{i} \\
& \dot{p}_{1}=-\partial H / \partial u^{1}=-(1 / c) \partial c / \partial u^{1}-(1 / 2) c^{2}\left(\partial g^{2 \jmath} / \partial u^{1}\right) p_{i} p_{j} \\
& \dot{p}_{2}=-\partial H / \partial u^{2}=-(1 / c) \partial c / \partial u^{2}-(1 / 2) c^{2}\left(\partial g^{\imath \jmath} / \partial u^{2}\right) p_{i} p_{j} \\
& \dot{\tau}=p_{1} \partial H / \partial p_{1}+p_{2} \partial H / \partial p_{2}=c^{2} g^{\imath \jmath} p_{i} p_{j}=1
\end{aligned}
$$

where ${ }^{-}$is a derivativation with respect to the ray parameter $t$.

Let $l$ be the arc length of the ray. Then from (2.10) and the eikonal equation (2.9) it follows immediately

$$
\begin{aligned}
l & =\int^{t}\left\{g_{\imath j}\left(d u^{\imath} / d t\right)\left(d u^{\jmath} / d t\right)\right\}^{1 / 2} d t=\int^{t}\left\{c^{4} g_{\imath j} g^{i k} g^{\jmath m} p_{k} p_{m}\right\}^{1 / 2} d t \\
& =\int^{t}\left\{c^{4} \delta_{\jmath}^{k} g^{\jmath m} p_{k} p_{m}\right\}^{1 / 2} d t=\int^{t}\left\{c^{4} g^{k m} p_{k} p_{m}\right\}^{1 / 2}=\int^{t} c d t,
\end{aligned}
$$

that is,

$$
d l / d t=c .
$$

Proposition. Assume all the rays are tangent to $\Gamma$. Let $l$ be the arc length along the ray measured from the point of tangency on $\Gamma$ and $n$ be the normal distance from the point on the ray to $\Gamma$. Then we have another formula for the effective curvature (1.1):

$$
K(s)=-d^{2} n /\left.d l^{2}\right|_{n=0}(s) .
$$

Proof. Put $\left(u^{1}, u^{2}\right)=(s, n)$. Then from (2.4) and (2.5) it can be easily seen that

$$
g_{11}=\left\{1-n K_{0}(s)\right\}^{2}, \quad g_{12}=g_{21}=0, \quad g_{22}=1 .
$$

In this case the eikonal equation (2.9) becomes

$$
(1 / 2)\left[c^{2}\left\{\left(1-n K_{0}(s)\right)^{-2}{p_{1}}^{2}+p_{2}{ }^{2}\right\}-1\right]=0 \quad\left(p_{1}=\partial_{s} \tau, p_{2}=\partial_{n} \tau\right) .
$$

So it follows from (2.10) that along the rays

$$
\begin{aligned}
\dot{s} & =c^{2}\left\{1-n K_{0}(s)\right\}^{-2} p_{1} \quad \dot{n}=c^{2} p_{2} \\
\dot{p}_{2} & =-(1 / c) \partial c / \partial n-c^{2}\left\{1-n K_{0}(s)\right\}^{-3} K_{0}(s) p_{1}{ }^{2} \\
& =-(1 / c) \partial c / \partial n-\left\{1-n K_{0}(s)\right\}^{-1}\left(1-c^{2}{p_{2}}^{2}\right) K_{0}(s) .
\end{aligned}
$$

Recalling that $p_{2}=\partial_{n} \tau=0$ at $n=0$ (the point of tangency) we have

$$
\dot{p}_{2}=-\partial_{n} c / c-K_{0}(s) \text { at } n=0 .
$$

Hence it is enough to show

$$
\left[d^{2} n / d l^{2}=\dot{p}_{2} \quad \text { at } \quad n=0 .\right.
$$

From (2.11) and (2.12) it follows 


$$
d n / d l=\dot{n} / \dot{l}=c p_{2}
$$

and

$$
d^{2} n / d l^{2}=d\left(c p_{2}\right) / d l=c^{-1} d\left(c p_{2}\right) / d t=c^{-1}\left\{\left(\partial_{n} c\right) \dot{n}+\left(\partial_{s} c\right) \dot{s}\right\} p_{2}+\dot{p}_{2},
$$

which proves (2.13).

Q.E.D.

Remark. The effective curvature here corresponnds to the minus of the generalized curvature for creeping waves. (see Lewis, Bleistein and Ludwig [2], p 318 (A2.3)) In [2], an asymptotic solution to the Helmholtz equation is constructed under the convexity condition $K<0$, which is called the creeping wave, while an asymptotic formula in [1] constructed under the concavity condition $K>0$ is called the whispering gallery wave.

Next we shall give the transformation invariant formula for the ray curvature at the point of tangency in the two dimensional Riemannian space.

THEOREM. Suppose that the boundary $\Gamma$ is given by $\varphi\left(u^{1} u^{2}\right)=0$ where $\varphi$ is a smooth function such that $\nabla \varphi \neq 0$ is the normal vector to $\Gamma$ pointing toward the domain $\Omega$. Assume all the rays are tangent $\Gamma$. Then the ray curvature at the point of tangency is given by

$$
K_{r}=-c^{-1}\left(\partial c / \partial u^{2}\right)\left(\partial \varphi / \partial u^{j}\right) g^{2 j}\left\{g^{m n}\left(\partial \varphi / \partial u^{m}\right)\left(\partial \varphi / \partial u^{n}\right)\right\}^{-1 / 2} .
$$

Proof. Let $l$ be the arc length of the ray. Then the unit tangent vector of the ray $d x / d l$ in $\left(u^{2}\right)$ coordinate is given by

$$
d x / d l=\left(d u^{1} / d l, d u^{2} / d l\right)
$$

which follows from the chain rule:

$$
\left(d x^{1} / d l, d x^{2} / d l\right)=\left(d u^{1} / d l\right) e_{1}+\left(d u^{2} / d l\right) e_{2} .
$$

Now we shall prove that the first derivative of the unit tangent vector of the ray $d^{2} x / d l^{2}$ in $\left(u^{2}\right)$ coordinate is given by

$$
d^{2} x / d l^{2}=\left(\Gamma_{{ }_{i k}}\left(d u^{2} / d l\right)\left(d u^{k} / d l\right)+d^{2} u^{1} / d l^{2}, \Gamma_{i k}^{2}\left(d u^{2} / d l\right)\left(d u^{k} / d l\right)+d^{2} u^{2} / d l^{2}\right)
$$

where $\Gamma_{i k}^{h}=g^{h j} \Gamma_{\imath j k}$ and $\Gamma_{\imath j k}=(1 / 2)\left(\partial g_{\imath j} / \partial u^{k}+\partial g_{j k} / \partial u^{\imath}-\partial g_{k \imath} / \partial u^{j}\right)$. Now we observe

$$
d^{2} x^{a} / d l^{2}=\left(\partial^{2} x^{a} / \partial u^{i} \partial u^{j}\right)\left(d u^{2} / d l\right)\left(d u^{j} / d l\right)+\left(\partial x^{a} / \partial u^{i}\right) d^{2} u^{2} / d l^{2} . \quad(a=1,2)
$$

Since

$$
\Gamma_{\imath j k}=\sum_{m=1}^{2}\left(\partial x^{m} / \partial u^{j}\right)\left(\partial^{2} x^{m} / \partial u^{k} \partial u^{i}\right) \text { and } g^{h \jmath}=\sum_{n=1}^{2}\left(\partial u^{h} / \partial x^{n}\right)\left(\partial u^{\jmath} / \partial x^{n}\right)
$$

we have 


$$
\begin{aligned}
\Gamma_{i k}^{h} & =\sum_{n=1}^{2}\left(\partial u^{h} / \partial x^{n}\right)\left(\partial u^{j} / \partial x^{n}\right) \sum_{m=1}^{2}\left(\partial x^{m} / \partial u^{j}\right)\left(\partial^{2} x^{m} / \partial u^{k} \partial u^{i}\right) \\
& =\sum_{m} \sum_{n}\left(\partial u^{h} / \partial x^{n}\right) \delta_{n}^{m}\left(\partial^{2} x^{m} / \partial u^{k} \partial u^{i}\right) \\
& =\left(\partial u^{h} / \partial x^{m}\right)\left(\partial^{2} x^{m} / \partial u^{k} \partial u^{i}\right),
\end{aligned}
$$

which leads to

$$
\left(\partial x^{a} / \partial u^{h}\right) \Gamma_{\imath \jmath}^{h}=\delta_{m}^{a}\left(\partial^{2} x^{m} / \partial u^{i} \partial u^{j}\right)=\partial^{2} x^{a} / \partial u^{i} \partial u^{\jmath} .
$$

Hence (2.16) is turned to

$$
d^{2} x^{a} / d l^{2}=\left(\partial x^{a} / \partial u^{h}\right) \Gamma_{{ }_{2 \jmath}}^{h}\left(d u^{2} / d l\right)\left(d u^{\jmath} / d l\right)+\left(\partial x^{a} / \partial u^{i}\right)\left(d^{2} u^{2} / d l^{2}\right), \quad(a=1,2)
$$

which proves (2.15).

In the second step we show

$$
\begin{aligned}
d^{2} x / d l^{2}= & \left(c\left(\partial c / \partial u^{k}\right) g^{1 j} g^{k m} p_{m} p_{j}-c^{-1}\left(\partial c / \partial u^{j}\right) g^{1 \jmath},\right. \\
& \left.c\left(\partial c / \partial u^{k}\right) g^{2 \jmath} g^{k m} p_{m} p_{j}-c^{-1}\left(\partial c / \partial u^{j}\right) g^{2 j}\right) .
\end{aligned}
$$

From (2.10) and (2.11) it follows

$$
d u^{2} / d l=\left(d u^{2} / d t\right)(d t / d l)=c g^{2 \jmath} p_{0}
$$

and

$$
\begin{aligned}
d^{2} u^{i} / d l^{2}= & \left\{d\left(c g^{\imath \jmath} p_{\jmath}\right) / d t\right\}(d t / d l) \\
= & \left\{\left(\partial c / \partial u^{k}\right)\left(d u^{k} / d t\right) g^{\imath \jmath} p_{j}+c\left(\partial g^{\imath \jmath} / \partial u^{k}\right)\left(d u^{k} / d t\right) p_{j}+c g^{2 j}\left(d p_{j} / d t\right)\right\} c^{-1} \\
= & c\left(\partial c / \partial u^{k}\right) g^{\imath \jmath} g^{k m} p_{\jmath} p_{m}+c^{2}\left(\partial g^{2 \jmath} / \partial u^{k}\right) g^{k m} p_{\jmath} p_{m} \\
& -c^{-1}\left(\partial c / \partial u^{j}\right) g^{\imath \jmath}-2^{-1} c^{2} g^{2 j}\left(\partial g^{k m} / \partial u^{j}\right) p_{k} p_{m} .
\end{aligned}
$$

Substituting them into the $\mu$ th component $(\mu=1,2)$ in $(2.15)$, we have

$$
\begin{aligned}
\Gamma_{i k}^{\mu}\left(d u^{2} / d l\right)\left(d u^{k} / d l\right)+\left(d^{2} u^{\mu} / d l^{2}\right)= & c^{2} \Gamma_{i k}^{\mu} g^{2 \jmath} g^{k m} p_{j} p_{m}+c\left(\partial c / \partial u^{k}\right) g^{\mu \jmath} g^{k m} p_{j} p_{m} \\
& +c^{2}\left(\partial g^{\mu \jmath} / \partial u^{k}\right) g^{k m} p_{\jmath} p_{m}-c^{-1}\left(\partial c / \partial u^{j}\right) g^{\mu \jmath} \\
& -2^{-1} c^{2} g^{\mu j}\left(\partial g^{k m} / \partial u^{j}\right) p_{k} p_{m} .
\end{aligned}
$$

Let $\left(g^{m h}\right)$ be the inverse matrix of $\left(g_{\imath \jmath}\right)$. Then differentiating the both sides of $g^{m h} g_{h \jmath}=\delta_{\jmath}^{m}$ with respect to $u^{k}$ we have

$$
\left(\partial g^{m h} / \partial u^{k}\right) g_{h j}+g^{m h}\left(\partial g_{h j} / \partial u^{k}\right)=0 .
$$

Multiplying the both sides by $g_{\imath m}$ implies

Hence we have

$$
\partial g_{\imath j} / \partial u^{k}=-g_{\imath m} g_{h j}\left(\partial g^{m h} / \partial u^{k}\right) .
$$

$$
\Gamma_{i k}^{\mu}=-2^{-1}\left\{g_{\imath m}\left(\partial g^{m \mu} / \partial u^{k}\right)+g_{h k}\left(\partial g^{\mu h} / \hat{\partial} u^{\prime}\right)-g^{\mu j} g_{k m} g_{h i}\left(\partial g^{m h} / \partial u^{j}\right)\right\}
$$

and 


$$
\begin{aligned}
c^{2} \Gamma_{i k}^{\mu} g^{\imath \jmath} g^{k m} p_{\jmath} p_{m}= & -2^{-1} c^{2} p_{\jmath} p_{m}\left\{\delta_{n}^{\jmath} g^{k m}\left(\partial g^{n \mu} / \partial u^{k}\right)+g^{\imath j} \delta_{h}^{m}\left(\partial g^{\mu h} / \partial u^{i}\right)\right. \\
& \left.-g^{\mu p} \delta_{n}^{m} \delta_{h}^{\jmath}\left(\partial g^{n h} / \partial u^{p}\right)\right\} \\
= & -c^{2} p_{\jmath} p_{m} g^{k m}\left(\partial g^{\jmath \mu} / \partial u^{k}\right)+2^{-1} c^{2} p_{\jmath} p_{m} g^{\mu p}\left(\partial g^{m \jmath} / \partial u^{p}\right) .
\end{aligned}
$$

Therefore we conclude

$$
\Gamma_{i k}^{\mu}\left(d u^{2} / d l\right)\left(d u^{k} / d l\right)+\left(d^{2} u^{\mu} / d l^{2}\right)=c\left(\partial c / \partial u^{k}\right) g^{\mu \jmath} g^{k m} p_{m} p_{j}-c^{-1}\left(\partial c / \partial u^{j}\right) g^{\mu \jmath} .
$$

This proves (2.17).

Since the covariant form of the normal vector to the boundary is $\left(\partial \varphi / \partial u^{1}\right.$, $\left.\partial \varphi / \partial u^{2}\right)$, the contravariant form of the normal vector is given by

$$
\left(g^{1 j} \partial \varphi / \partial u^{j}, g^{2 j} \partial \varphi / \partial u^{j}\right)
$$

The length of this vector is

$$
\begin{aligned}
\left\{g_{k \alpha} g^{k j}\left(\partial \varphi / \partial u^{j}\right) g^{\alpha \beta}\left(\partial \varphi / \partial u^{\beta}\right)\right\}^{1 / 2} & =\left\{\delta_{\alpha}^{j}\left(\partial \varphi / \partial u^{j}\right) g^{\alpha \beta}\left(\partial \varphi / \partial u^{\beta}\right)\right\}^{1 / 2} \\
& =\left\{g^{\alpha \beta}\left(\partial \varphi / \partial u^{\alpha}\right)\left(\partial \varphi / \partial u^{\beta}\right)\right\}^{1 / 2}
\end{aligned}
$$

Therefore the contravariant form of the unit normal vector pointing toward the domain $\Omega$ is given by

$$
\begin{aligned}
& \left(g^{1 j}\left(\partial \varphi / \partial u^{j}\right)\left\{g^{\alpha \beta}\left(\partial \varphi / \partial u^{\alpha}\right)\left(\partial \varphi / \partial u^{\beta}\right)\right\}^{-1 / 2},\right. \\
& \left.g^{2 j}\left(\partial \varphi / \partial u^{j}\right)\left\{g^{\alpha \beta}\left(\partial \varphi / \partial u^{\alpha}\right)\left(\partial \varphi / \partial u^{\beta}\right)\right\}^{-1 / 2}\right) .
\end{aligned}
$$

Now taking the inner product of the vectors (2.17) and (2.19) gives

$$
\begin{aligned}
K_{r}= & g_{i l}\left\{c\left(\partial c / \partial u^{k}\right) g^{\imath \jmath} g^{k m} p_{m} p_{j}-c^{-1}\left(\partial c / \partial u^{j}\right) g^{\imath j}\right\} \\
& \times g^{l n}\left(\partial \varphi / \partial u^{n}\right)\left\{g^{\alpha \beta}\left(\partial \varphi / \partial u^{\alpha}\right)\left(\partial \varphi / \partial u^{\beta}\right)\right\}^{-1 / 2} \\
= & \delta_{l}\left\{c\left(\partial c / \partial u^{k}\right) g^{k m} p_{m} p_{j}-c^{-1}\left(\partial c / \partial u^{j}\right)\right\} \\
& \times g^{l n}\left(\partial \varphi / \partial u^{n}\right)\left\{g^{\alpha \beta}\left(\partial \varphi / \partial u^{\alpha}\right)\left(\partial \varphi / \partial u^{\beta}\right)\right\}^{-1 / 2} \\
= & \left\{c\left(\partial c / \partial u^{k}\right) g^{k m} p_{m} p_{j}-c^{-1}\left(\partial c / \partial u^{j}\right)\right\} \\
& \times g^{\jmath n}\left(\partial \varphi / \partial u^{n}\right)\left\{g^{\alpha \beta}\left(\partial \varphi / \partial u^{\alpha}\right)\left(\partial \varphi / \partial u^{\beta}\right)\right\}^{-1 / 2} \\
= & \left\{c\left(\partial c / \partial u^{k}\right) g^{k m} p_{m} g^{\jmath n}\left(\partial \varphi / \partial u^{n}\right) p_{j}-c^{-1}\left(\partial c / \partial u^{j}\right)\left(\partial \varphi / \partial u^{n}\right) g^{{ }^{n}}\right\} \\
& \times\left\{g^{\alpha \beta}\left(\partial \varphi / \partial u^{\alpha}\right)\left(\partial \varphi / \partial u^{\beta}\right)\right\}^{-1 / 2} .
\end{aligned}
$$

Also, according to (2.10), the contravariant form of the tangent vector of the ray is given by

$$
\left(g^{1 \jmath} p_{\jmath}, g^{2 \jmath} p_{\jmath}\right)
$$

Therefore from the orthogonality of (2.18) and (2.21) at the point of tangency, it follows that

$$
0=g_{k n} g^{k \imath}\left(\partial \varphi / \partial u^{i}\right) g^{n_{\jmath}} p_{\jmath}=\delta_{n}^{\imath}\left(\partial \varphi / \partial u^{\imath}\right) g^{n^{\jmath}} p_{\jmath}=g^{n j}\left(\partial \varphi / \partial u^{n}\right) p_{\jmath} .
$$


Substituting this into the last equality in (2.20) we obtain the result. Q.E.D.

Since the transformation invariance of $g^{\imath j}\left(\partial c / \partial u^{\imath}\right)\left(\partial \varphi / \partial u^{j}\right)$ and $g^{m n}\left(\partial \varphi / \partial u^{m}\right)$ $\left(\partial \varphi / \partial u^{n}\right)$ in the right hand side of (2.14) follows from the property of contraction, we immediately obtain the following result.

Proposition. $K_{r}$ is an invariant under the coordinate transformation.

\section{REFERENCES}

[1] V.M. Babich And N.Y. Kirpichnikova, The Boundary Layer Method in Diffraction Problems, Springer Verlag, Berlin (1979).

[2] R.M. Lewis, N. Bleistein and D. Ludwig, Uniform Asymptotic Theory of Creeping Waves, Comm. Pure appl. Math., 20 (1967), 295-328.

Department of Mathematics

School of ScIENCE AND ENGINEERING

WASEDA UNIVERSITY

OKubo ShinjuKu

TOKYO 169 JAPAN 